\title{
Candidal endophthalmitis: a manifestation of systemic candidiasis
}

\author{
B.C.K. Patel, S.B. Kaye and L.H. Morgan \\ Manchester Royal Eye Hospital, Oxford Road, Manchester M13, UK.
}

\begin{abstract}
Summary: Two patients on total parenteral nutrition who developed endophthalmitis secondary to Candida albicans are described. Candidal endophthalmitis as a manifestation of systemic candidiasis is discussed and its early diagnosis by bedside fundal examination in patients at risk is stressed.
\end{abstract}

\section{Introduction}

Total parenteral nutrition (TPN) is being used increasingly for surgical in-patients and also as home parenteral nutrition (HPN), largely in patients with intestinal failure. Catheter sepsis is a well known complication in these patients but the risk of systemic candidiasis is generally underestimated. Systemic candidiasis is frequently unrecognized because of a lack of clinical signs. However, endophthalmitis is frequently a presenting feature of systemic candidiasis ${ }^{1}$ and its early detection is crucial to successful management. Clinical studies indicate that early detection improves the probability of therapeutic success with lower morbidity and improved patient survival. ${ }^{2}$ We present two cases of candidal endophthalmitis in patients receiving TPN.

\section{Case reports}

Case 1

A 56 year old woman presented with a large anterior abdominal wall abscess which was drained and intravenous metronidazole $500 \mathrm{mg}$ three times daily and cefotaxime $1 \mathrm{~g}$ twice daily commenced. Six days later, further surgical debridement was required and the doses of the metronidazole and cefotaxime were doubled. A subclavian line was inserted and intravenous hyperalimentation commenced. Twenty days after admission, cellulitis of the subclavian line site was noted. The line was removed and culture of the subclavian catheter grew Staphylococcus albus. Blood, urine and sputum cultures revealed no microorganisms.

Correspondence and present address: B.C.K. Patel, F.R.C.S., D.O., Moorfields Eye Hospital, City Road, London EC1 2PD, UK.

Accepted: 5 January 1987
The patient made steady progress and the intravenous antibiotics were changed to oral metronidazole and cephradine. Thirty-five days following presentation the patient complained of a painful, red right eye and blurred vision with the presence of 'floaters'. She had an uncorrected pin-hole visual acuity of $6 / 12$ in both eyes and had a right anterior and posterior uveitis. Fundal examination of the right eye revealed fluffy white cotton-ball like exudates nasal to the disc extending forwards from the retina into the vitreous with an associated haze in the surrounding posterior vitreous (Figure 1). The left eye was normal. Toxoplasma and cytomegalovirus antibody titres and repeat blood, urine and sputum cultures were all negative. In view of the history of parenteral nutrition

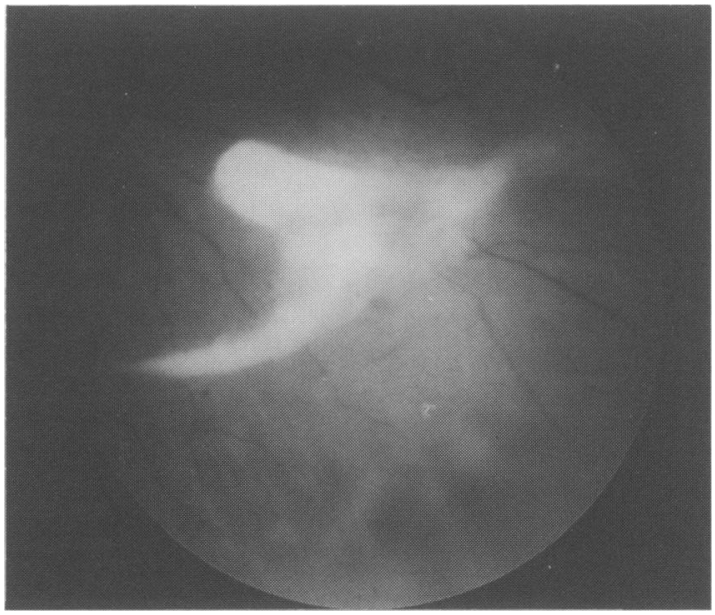

Figure 1 Case 1. White cotton-wool like fundal lesion with extension into the vitreous and surrounding vitreous haze. 
in a debilitated patient, the prolonged use of antibiotics and the typical fundal findings a diagnosis of fungal endophthalmitis was made and intravenous amphotericin B $40 \mathrm{mg} /$ day commenced. A right pars plana vitrectomy was carried out the same day and intravitreal amphotericin B $5 \mu \mathrm{g}$ injected. The vitreous aspirate specimen was concentrated by centrifugation and its culture revealed Candida albicans. The patient's therapy was changed to oral flucytosine $2 \mathrm{~g}$ four times daily and ketoconazole $200 \mathrm{mg} /$ day. This treatment was continued for 6 weeks and the chorioretinal lesions resolved completely. Her vision 2 months after vitrectomy was $6 / 18$ in the right eye.

\section{Case 2}

A 67 year old woman was commenced on TPN after small bowel resection for mesenteric ischaemia. She was commenced on intravenous metronidazole $500 \mathrm{mg}$ three times daily and cefuroxime $750 \mathrm{mg}$ twice daily. On the fourteenth day of parenteral nutrition she complained of an uncomfortable right eye. Her uncorrected vision with the pin-hole was $6 / 36$ in the right eye and $6 / 6$ in the left. Bedside fundal examination with pupillary dilatation with tropicamide $1 \%$ revealed several small yellow-white retinal lesions with minimal invasion of the vitreous body (Figure 2). A diagnosis of fungal endophthalmitis was made in view of her history, antibiotic usage with TPN and the clinical findings. Blood and sputum cultures were negative but a urine specimen revealed Candida albicans. The subclavian line was resited and culture of the subclavian catheter tip revealed no organisms. She was treated with parenteral amphotericin B $40 \mathrm{mg} /$ day for 8 days and then changed to oral flucytosine $2 \mathrm{~g}$ four

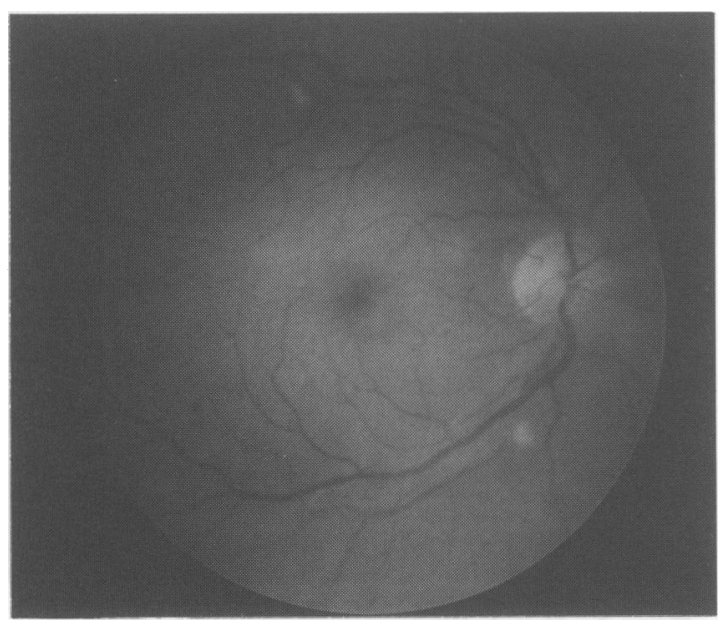

Figure 2 Case 2. Yellow-white retinal lesions with minimal extension into the vitreous. times daily and ketoconazole $200 \mathrm{mg} /$ day. This treatment was continued for 4 weeks and the retinal lesions regressed completely. Her vision 3 months after discharge was $6 / 36$ in the right eye.

\section{Discussion}

The diagnosis of disseminated candidiasis is made in $\frac{\bar{D}}{\bar{N}}$ the minority of cases. The incidence of candidaemia is $\overline{\mathbb{D}}$ increased in patients on intravenous hyperalimenta- $\stackrel{\circ}{\Omega}$ tion, ${ }^{3}$ haemodialysis, antibiotic therapy, after major $\tilde{N}$ surgery in intravenous heroin abuse $e^{4}$ and in the $\overrightarrow{0}$ immunocompromised patient. Earlier reports sugges- $\vec{\overrightarrow{ }}$ ted that most episodes of candidaemia resolve spon- $\vec{\omega}$ taneously. ${ }^{5}$ However, even transient candidaemia may $\overline{0}$ not be benign and disseminated candidiasis may follow. Although clinical indicators of disseminated candidiasis are lacking, it has been shown that $78 \%$ of $\omega$ patients with candidal endophthalmitis have dissemin- $\nexists$ ated candidiasis and that candidal endophthalmitis is $\overrightarrow{i r}$ likely to occur in $30-37 \%$ of patients with can- O) didaemia. $^{6}$

The patient with disseminated candidiasis presents with headaches, malaise and rigors and the common- $c$ est metastatic manifestation is the presence of tender nodules on the scalp. ${ }^{7}$ Candidal endophthalmitis is $\overrightarrow{0}$ another important metastatic manifestation and the finding of this ocular disease in any patient belonging to any of the risk groups is presumptive evidence fo the presence of disseminated candidiasis. ${ }^{2}$ The contmonest ocular symptoms of candidal endophthalmitis are pain, photophobia, blurred vision and floaters although in the early stages these patients may be asymptomatic. The earliest fundal lesion is a $\overrightarrow{\bar{O}}$ chorioretinitis which presents as small yellow-white lesions (Figure 2) which may be confused with cottonwool spots, hard exudates of hypertensive retinopathy, and chorioretinitis caused by other infections like toxoplasmosis, histoplasmosis and cytomegalovirus. Extension of the infection and its associated inflammatory response into the vitreous body produces the more typical lesions described as 'puff balls ${ }^{8}$ (Figure 1). Sometimes these lesions are joined by strands giving the appearance of a 'string of pearls'. These vitreal lesions make the vitreous cloudy owing $\frac{D}{0}$ to inflammatory cells and increased protein content of the vitreous. Other ocular findings include anterior $N$ uveitis, scleritis, intraretinal haemorrhages, iris abs- $N$ cesses and papillitis. Although spontaneous resolution of candidal endophthalmitis has been reported, ${ }^{9}$ in the ${ }^{\omega}$ absence of effective treatment, retinal necrosis and vitreal organization occur with tractional retinal deta- $\stackrel{0}{\odot}$ chment leading to phthisis bulbi with complete loss of $\stackrel{\mathscr{D}}{\rightarrow}$ visual function ${ }^{10}$ and in one large series, $50 \%$ of patients with candidal endophthalmitis died. ${ }^{2}$

Various treatment regimes are in use but parenteral 
amphotericin B is the most frequently used anti-fungal agent. This, together with intravitreal amphotericin B have been well shown to be an effective mode of treatment for fungal endophthalmitis ${ }^{8}$ although retinal toxicity is a risk with higher doses of intravitreal amphotericin B. " Recently, two new antifungal agents, flucytosine and ketoconazole have been available. These have the advantage of allowing oral therapy and their combined use, as used for the treatment of both these cases, to prevent the emergence of resistant candidal organisms, has been advocated. ${ }^{12}$ Successful treatment results in the diminution of the vitreal lesions and the inflammatory cell infiltrate and the chorioretinal lesions heal, occasionally with localized gliotic scars. Sometimes, resolution of the vitreal inflammatory process is accompanied by fibrosis with localized macular traction and retinal detachment.

The exact role of vitrectomy has not been established but is generally recommended when progression of intraocular infection occurs despite systemic antifungal therapy. ${ }^{13}$ Vitrectomy removes the vitreous focus of infection which serves as a scaffold for fibrosis and also possibly enhances the ocular penetration of systemic therapy. Vitrectomy has also been advocated for diagnostic purposes and this is particularly useful when the specimen obtained is concentrated by centrifugation prior to culture. ${ }^{14}$ However, vitrectomy is not without risk and intravitreal injection of antifungal agents can lead to retinal toxicity and macular fibrosis. In case 1 , vitrectomy was carried out to reduce

\section{References}

1. Fishman, L.S., Griffin, J.R., Sapico, F.L. \& Hecht, R. Hematogenous Candida endophthalmitis: a complication of candidemia. N Engl J Med 1972, 286: 675-681.

2. Edwards, J.E.Jr., Foos, R.Y., Montgomerie, J.Z. \& Guze, L.B. Ocular manifestations of Candida septicemia: review of seventy-six cases of hematogenous Candida endophthalmitis. Medicine (Baltimore) 1974, 53:47-75.

3. Curry, C.R. \& Quie, P.G. Fungal septicemia in patients receiving parenteral hyperalimentation. $N$ Eng. $J$ Med 1971, 285: 1221-1225.

4. Servant, J.B., Dutton, G.N., Ong-tone, L., Barrie, T. \& Davey, C. Candidal endophthalmitis in Glaswegian heroin addicts: Report of an epidemic. Trans Ophthalmol Soc UK 1985, 104: 297-308.

5. Ellis, C.A. \& Spival, M.L. The significance of candidemia. Ann Intern Med 1967, 67: 511-522.

6. Parke, D.W., Jones, D.B. \& Gentry, L.O. Endogenous endophthalmitis among patients with candidemia. Ophthalmology 1982, 89: 789-796.

7. Dally, S. \& Thomas, G. Loss of hair, blindness and skin rash in heroin addicts. Vet Hum Toxicol 1982, (Suppl): 62-63.

8. Perraut, L.E. Jr., Perraut, L.E., Bleiman, B. \& Lyons, J. Successful treatment of Candida albicans endophthalmitis with intravitreal amphotericin B. Arch Ophthalmol the infective burden in the eye as the lesions were advanced with marked vitreal invasion. The aspirate also confirmed the clinical impression and hence allowed the use of specific appropriate treatment. In case 2 , the lesions were at an early stage with chorioretinal involvement but minimal invasion of the vitreous body and hence no vitrectomy was carried out.

Systemic candidiasis and candidal endophthalmitis may occur in the absence of positive blood cultures as in both these cases. In one prospective study, $10.6 \%$ of patients on TPN developed candidal endophthalmitis and of these $50 \%$ had negative multiple blood cultures. ${ }^{15}$ The diagnosis of systemic candidiasis may therefore only be suspected when the patient presents with symptoms and signs of candidal endophthalmitis. The fundal findings of candidal endophthalmitis are easily detectable by bedside direct ophthalmoscopy through the dilated pupil and early detection of such lesions is important for two main reasons. Firstly, as candidal endophthalmitis has a strong association with disseminated candidiasis, systemic anti-fungal therapy needs to be instituted to prevent the high mortality associated with this condition. ${ }^{16}$ Secondly, prompt treatment of these early lesions may avert the poor visual outcome that would otherwise occur. ${ }^{10}$

We believe that all patients with suspected systemic candidiasis and especially those on TPN should have fundal examination after pupillary dilatation even if no ocular symptoms are present as awareness of fungal endophthalmitis may lead to salvage of useful vision.

1981, 99: $1565-1567$.

9. Dellon, A.L., Stark, W.J. \& Chretien, P.B. Spontaneous resolution of endogenous Candida endophthalmitis complicating intravenous hyperalimentation. Am J Ophthalmol 1975, 79: 648-654.

10. Griffin, J.R., Pettit, T.H., Fishman, L.S. \& Foos, R.Y. Blood-borne Candida endophthalmitis. Arch Ophthalmol 1973, 89: 450-456.

11. Souri, E.N. \& Green, R.W. Intravitreal amphotericin B toxicity. Am J Ophthalmol 1974, 78: 77-81.

12. Medoff, G. \& Kobayashi, G.S. Strategies in the treatment of systemic fungal infections. N Engl J Med 1980, 302: $145-155$.

13. Snip, R.C. \& Michels, R.G. Pars plana vitrectomy in the management of endogenous Candida endophthalmitis. Am J Ophthalmol 1976, 82: 699-704.

14. Aguilar, G.L., Blumenkrantz, M.S., Egbert, P.R. \& McCulley, J.P. Candida endophthalmitis after intravenous drug abuse. Arch Ophthalmol 1979, 97: 96-100.

15. Henderson, D.K. \& Remington, J.S. Haematogenous candidal endophthalmitis in surgical patients receiving parenteral fluids. Clin Res 1979, 27: 41A.

16. Klein, J.J. \& Watanakunakorn, C. Hospital acquired fungemia. Its natural course and clinical significance. $\mathrm{Am}$ J Med 1979, 67: 51-57. 